\title{
Sliding mode stabilization of the current profile in Tokamak plasmas
}

\author{
Oumar Gaye, Emmanuel Moulay, Sylvain Brémond, Laurent Autrique, Rémy Nouailletas and Yury Orlov
}

\begin{abstract}
This paper deals with the robust stabilization of the spatial distribution of the tokamak plasmas current profile using a sliding mode feedback control approach. The control design is based on the $1 D$ resistive diffusion equation of the magnetic flux that governs the plasma current profile evolution. The feedback control law is derived in the infinite dimensional setting without spatial discretisation. Numerical simulations are provided and the tuning of the controller parameters that would reject uncertain perturbations is discussed.
\end{abstract}

\section{INTRODUCTION}

The fossil fuels (oil, gas, coal) represent for approximately $85 \%$ of the worldwide sources of primary energy today. But they should run out some tens of years and they are responsible for a climate change via the contribution in the greenhouse effect of the $\mathrm{CO}_{2}$ generated by their combustion.

The controlled thermonuclear fusion is one of the options being studied in order to eventually provide an answer. Its main assets are to be a potential inexhaustible and safe source of energy because the reserves in nuclear fuel are plenty (Deuterium can be extracted from sea water, and Lithium, that has to be used to generate Tritium, can be found in continental crust) and because there is no risk of runaway reaction nor long lasting radioactive waste. The key world project in the domain, ITER [1], is led by seven partners (Europe, United States of America, Japan, China, India, South Korea, Russia) accounting for one half of the world population. The main objective of the ITER project is to demonstrate the scientific feasibility of thermonuclear fusion.

Several conditions have to be met to produce fusion reactions [2]: the fuels have to be heated up to very high temperature (around 100 millions degrees) in order to overcome the electrostatic potential barrier between positively charged nucleus. To reach such a temperature, the ionized gas or plasma must be confined, for example by magnetic

Manuscript received March 2, 2011. This work was supported in part by the CEA (Commissariat á l'Energie Atomique) and the French région Pays de Loire.

O. Gaye is with LISA (EA 4094) - Université d'Angers, 62 avenue Notre Dame du Lac, 49000 Angers - France oumar.gayedetud.univ-angers. fr

E. Moulay is with Xlim-SIC (UMR CNRS 6172), Université de Poitiers - Bât. SP2MI, Bvd Marie et Pierre Curie BP 30179, 86962 Futuroscope Chasseneuil Cedex - France emmanuel.moulay@univ-poitiers.fr

S. Brémond is with CEA/DSM/IRFM CEA-Cadarache, 13108 Saint Paul Lez Durance - France Sylvain. BREMOND@cea. fr

L. Autrique is with LISA (EA 4094) - Université d'Angers, 62 avenue Notre Dame du Lac, 49000 Angers - France laurent . autriqueduniv-angers. fr

R. Nouailletas is with CEA/DSM/IRFM CEA-Cadarache, 13108 Saint Paul Lez Durance - France Remy . NOUAILLETAS@ cea . fr

Y. Orlov is with CICESE Research Center, Km 107 Carr. TijuanaEnsenada, Ensenada, B.C. 22860 - Mexico yorlov@cicese.mx

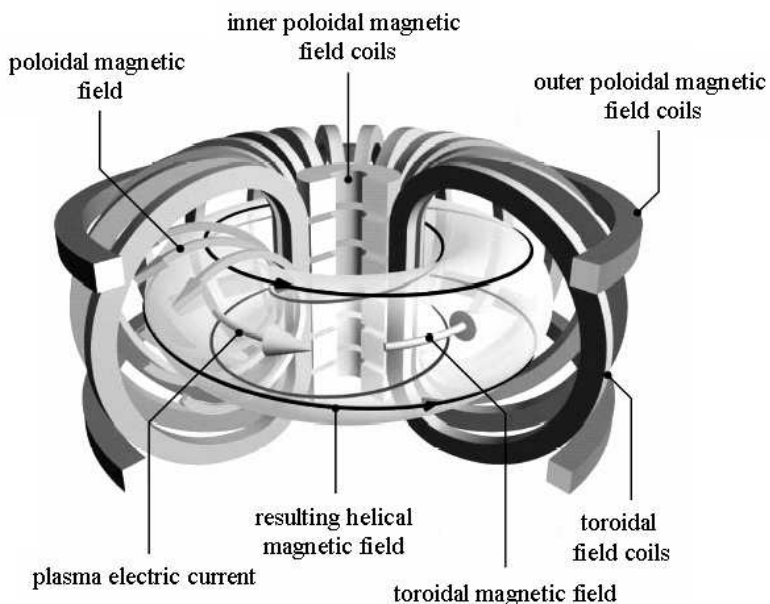

Fig. 1. Tokamak magnetic configuration

confinement which seems to be the most promising way. This magnetic confinement is obtained in a tokamak (see [3]) facilities (Cf. Fig. 1) by superimposing different electric currents in a torus-like configuration device, including a high current, of the order of the MegaAmpere, within the plasma itself.

This plasma current can be produced by inductive means, in particular at the beginning of the plasma pulses, and by non-inductive means through the injection of fast particles and /or waves. The 1D radial profile of this plasma current, via the so-called safety factor profile, is known to be a key parameter for tokamak plasma performance. It indeed plays a crucial role in the global magnetohydrodynamic (MHD) stability of plasma experiments. Moreover, it has been observed that some specific profiles may generate some enhanced confinement of the plasma energy. It is obvious that such profiles are very attractive and may at the end reduce the size and cost of future fusion reactors. Several approaches have been developed regarding the control of tokamak plasma current profile. The control of one single shape parameter, based on Single Input - Single Output (SISO) semi-empirical approaches, has been performed experimentally (e.g plasma internal inductance control [4] or non-inductive current drive profile width [5]). But this is clearly not enough to match the main requirements of MHD stability and/or internal transport barrier issues in advanced tokamak scenarios. The control of the safety factor profile in a few number of points, based on a Multi input - Multi Output (MIMO) approach, was developed using linear models identified from experimental data [6]. It was experimentally tested but showed severe limitations 


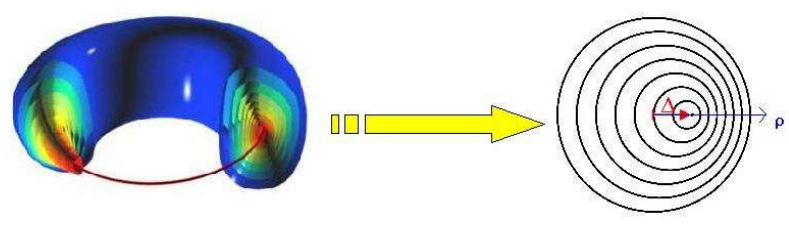

Fig. 2. 1D geometry of the simplified formulation of the problem

in terms of robustness. Some other recent approaches can also be found in [7]-[9]. The approach proposed in this paper is developed in the infinite dimensional setting without space discretisation. This discretization is required only at the controller implementation stage.

The paper is organized as follows. In the section II we introduce the distributed control model, based on the 1D resistive diffusion Partial Differential Equation (PDE) of the magnetic flux that governs the plasma current profile evolution. Details are given on the transformation of the PDE that are required in order to prepare the control design. Section III is devoted to the construction of a control law. Finally, simulation results are provided in section IV and conclusion remarks in section $\mathrm{V}$.

\section{CONTROL MODEL MATHEMATICAL ANALYSIS}

Provided usual assumptions (axisymetry, MHD equilibrium, averaging over the magnetic surfaces, cylindrical approximation, etc. see [10] and [11]), evolution of the plasma safety factor $q$ (current profile) can be obtained by solving the following 1D PDE:

$$
\begin{aligned}
& \left\{\begin{array}{l}
\frac{\partial \psi}{\partial t}=\frac{\eta_{\|}}{\mu_{0} a^{2}} \frac{1}{x} \frac{\partial}{\partial x}\left(x \frac{\partial \psi}{\partial x}\right)+\eta_{\|} R_{0} j_{n i} ; \\
\left.\frac{\partial \psi}{\partial x}\right|_{x=0}=0, \quad \frac{\partial \psi}{\partial t}(t, 1)=-V_{0}(t) .
\end{array}\right. \\
& q=-\frac{a^{2} x B_{0}}{\frac{\partial \psi}{\partial x}} \\
& j_{T}=-\frac{1}{\mu_{0} R_{0} a^{2} x} \frac{\partial}{\partial x}\left(x \frac{\partial \psi}{\partial x}\right)
\end{aligned}
$$

where $x \in(0,1)$ is the $1 D$ (radial) profile coordinate, $\psi(t, x)$ the magnetic flux, $R_{0}$ and $a$ are respectively the major and minor radius of the plasma boundary (assumed to be fixed), $\mu_{0}$ the permeability of vacuum, $\eta_{\|}(t, x)$ the parallel resistivity of the plasma, $j_{n i}(t, x)$ the non-inductive current density and $V_{0}(t)$ the plasma loop voltage, $B_{0}$ the toroidal magnetic field at $R_{0}$ and $j_{T}$ is the total current density. $q$ is the safety factor to be controlled. In the present investigation, the resistivity $\eta_{\|}(t, x)$ is assumed to be lower and upper bounded by some positive constants $\eta_{1}$ and $\eta_{2}$. Moreover, we consider that $\eta_{\|}(t, x)$ is available for feedback purposes through some on-line estimation (basically from electronic temperature measurements).

Our control objective is to track a desired safety factor profile which does not depend directly on $\psi$ but which depends on its spatial derivative $\frac{\partial \psi}{\partial x}$.
In order to deal with homogeneous boundary conditions, let us introduce the following state transformation:

$$
\psi_{r}(t, x)=\psi(t, x)-\psi(t, 1) .
$$

The state equation (1), rewritten in terms of $\psi_{r}$, reduces to

$$
\left\{\begin{array}{l}
\frac{\partial \psi_{r}}{\partial t}=\frac{\eta_{\|}}{\mu_{0} a^{2}} \frac{1}{x} \frac{\partial}{\partial x}\left(x \frac{\partial \psi_{r}}{\partial x}\right)+\eta_{\|} R_{0} j_{n i}+V_{0}(t) \\
\left.\frac{\partial \psi_{r}}{\partial x}\right|_{x=0}=0, \quad \psi_{r}(t, 1)=0
\end{array}\right.
$$

Then, let us introduce the error variable

$$
\phi(t, x)=\psi_{r}(t, x)-\psi_{r}^{\infty}(x)
$$

with respect to the target $\psi_{r}^{\infty}(x)$ which we intend to reach in the Sobolev space

$$
W^{1,2}(0,1)=\left\{\psi \in L^{2}(0,1): \frac{\partial \psi}{\partial x} \in L^{2}(0,1)\right\}
$$

of differentiable functions, whose spatial derivative is square integrable on the interval $(0,1)$. Then the error variable is governed by:

$$
\left\{\begin{array}{c}
\frac{\partial \phi}{\partial t}=\frac{\eta_{\|}}{\mu_{0} a^{2}} \frac{1}{x} \frac{\partial}{\partial x}\left(x \frac{\partial \phi}{\partial x}\right)+\frac{\eta_{\|}}{\mu_{0} a^{2}} \frac{1}{x} \frac{\partial}{\partial x}\left(x \frac{\partial \psi_{r}^{\infty}}{\partial x}\right) \\
+\eta_{\|} R_{0} j_{n i}+V_{0}(t) ; \\
\left.\frac{\partial \phi}{\partial x}\right|_{x=0}=-\left.\frac{\partial \psi_{\infty}}{\partial x}\right|_{x=0}, \quad \phi(t, 1)=-\psi_{\infty}(1) .
\end{array}\right.
$$

In order to deal with the regular term $\frac{1}{x} \frac{\partial}{\partial x}\left(x \frac{\partial \psi_{r}^{\infty}}{\partial x}\right)$ in (5) and homogeneous boundary conditions we assume that

$$
\begin{gathered}
\lim _{x \rightarrow 0}\left|\frac{1}{x} \frac{\partial \psi_{r}^{\infty}}{\partial x}\right|<+\infty ; \\
\left.\frac{\partial \psi_{r}^{\infty}}{\partial x}\right|_{x=0}=0 ; \quad \psi_{r}^{\infty}(1)=0 .
\end{gathered}
$$

These assumptions lead to the system with homogeneous boundary conditions:

$$
\left\{\begin{array}{c}
\frac{\partial \phi}{\partial t}=\frac{\eta_{\|}}{\mu_{0} a^{2}} \frac{1}{x} \frac{\partial}{\partial x}\left(x \frac{\partial \phi}{\partial x}\right)+\frac{\eta_{\|}}{\mu_{0} a^{2}} \frac{1}{x} \frac{\partial}{\partial x}\left(x \frac{\partial \psi_{r}^{\infty}}{\partial x}\right) \\
+\eta_{\|} R_{0} j_{n i}+V_{0}(t) ; \\
\left.\frac{\partial \phi}{\partial x}\right|_{x=0}=0, \quad \phi(t, 1)=0 .
\end{array}\right.
$$

Since

$$
\frac{\eta_{\|}}{x} \frac{\partial}{\partial x}\left(x \frac{\partial \phi}{\partial x}\right)=\frac{\partial}{\partial x}\left(\eta_{\|} \frac{\partial \phi}{\partial x}\right)+\left(\frac{\eta_{\|}}{x}-\frac{\partial \eta_{\|}}{\partial x}\right) \frac{\partial \phi}{\partial x}
$$

the equation (8) with the singular term $\frac{1}{x}$ can be brought into the regular form without singularities by applying the following feedback transformation

$$
\eta_{\|} R_{0} j_{n i}=\eta_{\|} u+v
$$

with a virtual control input $u$ and

$$
\begin{array}{r}
v=-V_{0}(t)+\frac{1}{\mu_{0} a^{2}}\left(-\frac{\eta_{\|}}{x}+\frac{\partial \eta_{\|}}{\partial x}\right) \frac{\partial \psi}{\partial x} \\
-\frac{1}{\mu_{0} a^{2}}\left(-\frac{\eta_{\|}}{x}+\frac{\partial \eta_{\|}}{\partial x}\right) \frac{\partial \psi_{r}^{\infty}}{\partial x}-\frac{\eta_{\|}}{\mu_{0} a^{2}} \frac{1}{x} \frac{\partial}{\partial x}\left(x \frac{\partial \psi_{r}^{\infty}}{\partial x}\right)
\end{array}
$$


is deduced from the state transformations (2) and (4). Then substituting (10) subject to (11) into (8) yields

$$
\left\{\begin{array}{l}
\frac{\partial \phi}{\partial t}=\frac{\partial}{\partial x}\left(\frac{\eta_{\|}}{\mu_{0} a^{2}} \frac{\partial \phi}{\partial x}\right)+\eta_{\|} u \\
\left.\frac{\partial \phi}{\partial x}\right|_{x=0}=\phi(t, 1)=0
\end{array}\right.
$$

The resulting equation is a standard parabolic equation in the Sobolev space:

$$
\phi \in H=\left\{\varphi \in W^{1,2}(0,1):\left.\frac{\partial \varphi}{\partial x}\right|_{x=0}=\left.\varphi\right|_{x=1}=0\right\}
$$

of the square integrable functions subject to the boundary conditions corresponding to the boundary value problem (12) and equipped with the norm

$$
\|\varphi\|=\sqrt{\int_{0}^{1}\left(\frac{\partial \varphi}{\partial x}\right)^{2} d x}
$$

The operator

$$
A \phi=\frac{\partial}{\partial x}\left(\frac{\eta_{\|}}{\mu_{0} a^{2}} \frac{\partial \phi}{\partial x}\right)
$$

that appears in the right-hand side of the PDE (12), is defined on the domain $D(A)=\left\{\phi \in H: \frac{\partial^{2} \varphi}{\partial x^{2}} \in L^{2}(0,1)\right\}$. This operator is recognized as a Sturm-Liouville operator [12] and its spectrum consists of the discrete values

$$
\lambda_{k}=-\frac{1}{\mu_{0} a^{2}}\left(\frac{\left(k-\frac{1}{2}\right) \pi}{\int_{0}^{1} \frac{d s}{\eta_{\|}(s)}}\right)^{2} \frac{1}{\eta_{\|}(x)}, k=1,2, \ldots
$$

that correspond to the following eigenfunctions

$$
\phi_{k}^{0}(x)=\cos \left(\left(k-\frac{1}{2}\right) \pi \frac{\int_{0}^{x} \frac{d s}{\eta_{\|}(s)}}{\int_{0}^{1} \frac{d s}{\eta_{\|}(s)}}\right)
$$

The Sturm-Liouville operator $A$ generates an exponentially stable semigroup. Moreover the system (12) admits continuous solution and is null controllable if $u$ belongs to $L^{2}$ (see [13], [14] for details). It implies that the system (12) is exponentially stabilizable (see [15, Theorem 4.12, p. 407]). Consequently, we can apply the sliding mode strategy developed in [16].

\section{SLIDING MODE CONTROLLER}

The problem of the control of partial differential equations (PDEs) is an active area of research [17]- [22], but very few constructive method are available. For robust stabilization, a sliding mode strategy has nevertheless recently been developed [16].

The sliding mode control approach, developed in [16] for infinite dimensional systems, is further adapted to be applied to control problem. The virtual control input $u$ is designed as follows:

$$
u(\phi)=-\left(\mathscr{N}+L \sqrt{\sum_{k=0}^{k=N}\left(P^{k}(\phi)\right)^{2}}\right) \operatorname{sign}\left(\sum_{k=0}^{k=N} c_{k} P^{k}(\phi)\right)
$$

where $C=\left(c_{k}\right)_{k=0}^{N}$ is the sliding surface, $\mathscr{N}$ is determined to reject the disturbances, $L$ is determined to ensure the Lyapunov stability and $N$ is the number of projections $P^{k}$ on the eigenfunction of the operator $A$ defined by

$$
P^{k}(\phi)=\frac{1}{\left\|\phi_{k}^{0}\right\|} \int_{0}^{1} \phi(t, x) \phi_{k}^{0}(x) d x, k=1,2, \ldots
$$

$C$ satisfies the following relation (see [16])

$$
\dot{x}_{1}=\left[A_{1}-B_{1}\left(C B_{1}\right)^{-1} C A_{1}\right] x_{1}
$$

where $x_{1}=\left(P^{0} \phi(t, .), \ldots, P^{N} \phi(t, .)\right)^{T}$ is the projection of $\phi$ on the first $N+1$ eigenfunctions of operator $A$ and where

$$
\begin{aligned}
& A_{1}=\operatorname{diag}\left\{\lambda_{k}\right\}_{k=0}^{N} \in \mathbb{R}^{N+1 \times N+1}, \\
& B_{1}=\left(P^{0} \eta_{\|}, P^{1} \eta_{\|}, \ldots, P^{N} \eta_{\|}\right)^{T} .
\end{aligned}
$$

We set

$$
A_{c}=A_{1}-B_{1}\left(C B_{1}\right)^{-1} C A_{1}
$$

and we have

$$
C A_{c}=C A_{1}-C B_{1}\left(C B_{1}\right)^{-1} C A_{1}=0 \Leftrightarrow A_{c}^{T} C^{T}=0 .
$$

Consequently $C^{T}$ is a eigenvector of $A_{c}^{T}$ associated to the eigenvalue $\lambda=0$. Now we set

$$
K=\left(C B_{1}\right)^{-1} C A_{1} \text {. }
$$

From (16), we obtain

$$
\dot{x}_{1}=\left[A_{1}-B_{1} K\right] x_{1},
$$

Choosing $K$ such that $A_{c}=A_{1}-B_{1} K$ has a first eigenvalue equal to zero while other eigenvalues are strictly negative in order to ensure the stability of the system (19). In order to determine $L$, we consider the system in $x_{1}$ without disturbance $(\mathscr{N}=0)$

$$
\dot{x}_{1}=A_{1} x_{1}+B_{1} u
$$

and the Lyapunov function

$$
V=\frac{1}{2} S^{2}>0, \quad \text { with } S=C x_{1} .
$$

It follows that

$$
\begin{aligned}
\frac{d V}{d t} & =S \dot{S}=C x_{1} C\left(A_{1} x_{1}+B_{1} u\right) \\
& =C x_{1} C A_{1} x_{1}-C x_{1} C B_{1} L\left\|x_{1}\right\| \operatorname{sign}(S) .
\end{aligned}
$$

We have $\dot{V}<0$ if and only if.

$$
C x_{1} C A_{1} x_{1}<C B_{1} L\left\|x_{1}\right\|\left|C x_{1}\right| .
$$

We know that

$$
C x_{1} C A_{1} x_{1} \leq\left|C x_{1}\right|\left\|C A_{1}\right\|\left\|x_{1}\right\|
$$

and

$$
C B_{1} L\left\|x_{1}\right\|\left|C x_{1}\right| \leq\left|C x_{1}\right|\left\|C B_{1}\right\| L\left\|x_{1}\right\| .
$$

In order to have $\dot{V}<0$ it is sufficient to have

$$
\left|C x_{1}\right|\left\|C A_{1}\right\|\left\|x_{1}\right\|<C B_{1} L\left\|x_{1}\right\|\left|C x_{1}\right| .
$$

Then from (24) and (25), we obtain

$$
L>\frac{\left\|C A_{1}\right\|}{\left\|C B_{1}\right\|} \text {. }
$$




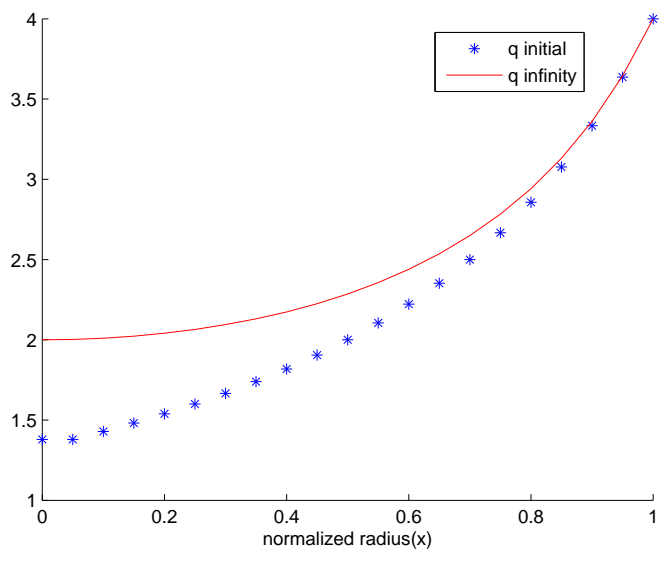

Fig. 3. The curve $q$ initial and $q_{\infty}$ (target of $q$ )

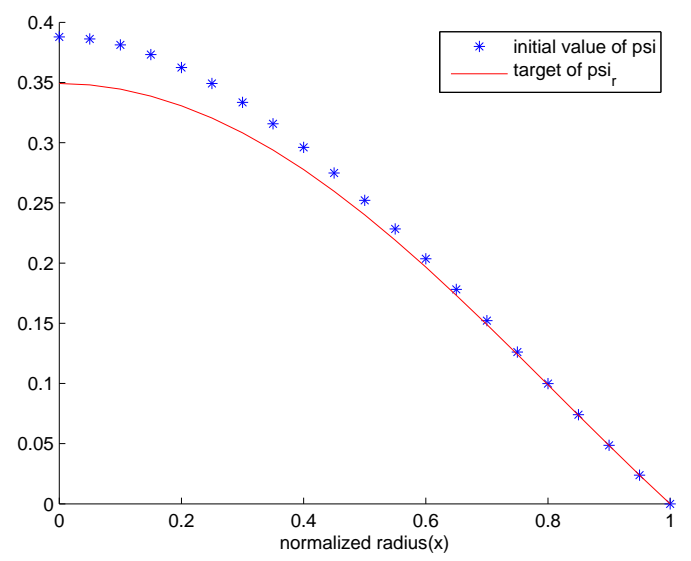

Fig. 4. The curve $\psi$ initial and $\psi_{r}^{\infty}$ (target of $\psi_{r}$ )

We see that the constant $L$ is lower bounded. Moreover, the proposed control law (15), specified with (12), rejects any additive external disturbances

$$
\begin{gathered}
\alpha(t, x)=\eta_{\|} h(t, x) \\
\left\{\begin{array}{l}
\frac{\partial \phi}{\partial t}=\frac{\partial}{\partial x}\left(\frac{\eta_{\|}}{\mu_{0} a^{2}} \frac{\partial \phi}{\partial x}\right)+\eta_{\|} u+\alpha(t, x) ; \\
\left.\frac{\partial \phi}{\partial x}\right|_{x=0}=\phi(t, 1)=0 .
\end{array}\right.
\end{gathered}
$$

with a priori known upper bounds $\mathscr{H}>0$ provided that

$$
\|h\| \leq \mathscr{H}<\mathscr{N} .
$$

Summarizing the following result is obtained.

Theorem 1: Consider the error system (12) with the assumptions above. Let it be driven by the sliding mode controller (15). Then the closed-loop system is finite time stable in the state space $H$. The finite time stability remains in force even if system (29) is additively affected by an external disturbance (28) satisfying (30).
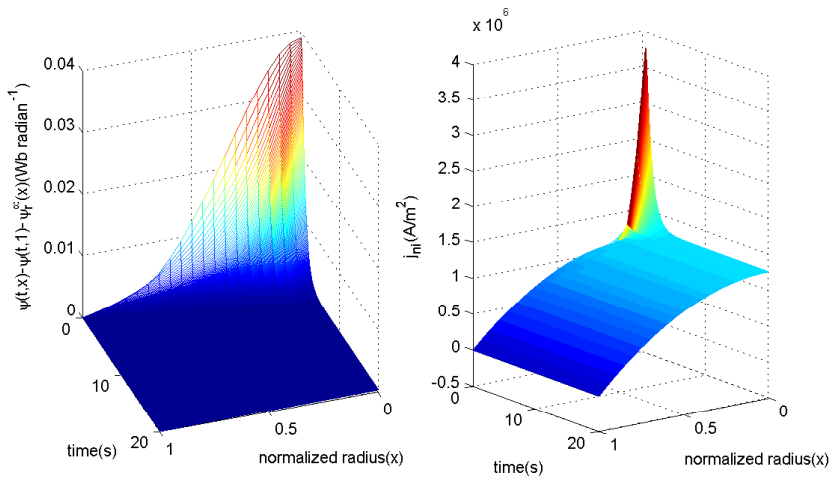

Fig. 5. Evolution of the flux profile error variable $\phi$ and the control $j_{n i}$ : $\mathscr{N}=0, L=10$ and $N=10$

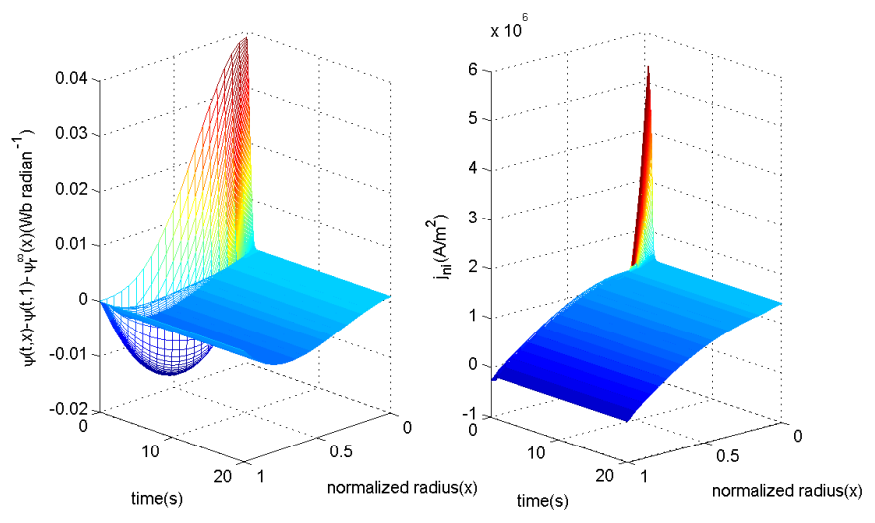

Fig. 6. Evolution of the flux profile error variable $\phi$ and the control $j_{n i}$ : $\mathscr{N}=7.10^{5}, L=10$ and $N=10$ with a disturbance $20 \%$ of $j_{\infty}$

\section{NUMERICAL SIMULATION}

\section{A. Test Cases Definition}

We consider the relevant test case where we want to reach a target safety factor profile presented in Fig. 3 : at $x=0$, the initial minimum value is $q(t, 0)=4 / 3$ and we would like to obtain a minimum value: $q(\infty, 0)=2$. At the plasma boundary $x=1$, the safety factor is fixed (this situation corresponds to a fixed total plasma current). The detailed target, initial safety factor profiles and corresponding magnetic flux profiles (with magnetic flux equal to zero at the plasma boundary $x=1$ ) are given in Figs. 3 and 4. The closed loop control simulations were performed with and without a disturbance (see (28)). The disturbance was scaled to $20 \%$ of the non inductive current profile $j_{\infty}(x)=j_{n i}(\infty, x)$ corresponding to the desired steady state, in order to take into account effects of model uncertainties and disturbances. For the purpose of the simulation, the plasma resistivity $\eta_{\|}(t, x)$ is assumed to depend only on the space variable as a second order polynomial with typical Tore Supra values $\left(10^{-8}\right.$ Ohms.m at the plasma center $x=0$ with two order of magnitude greater value at the plasma boundary $x=1$ ). 


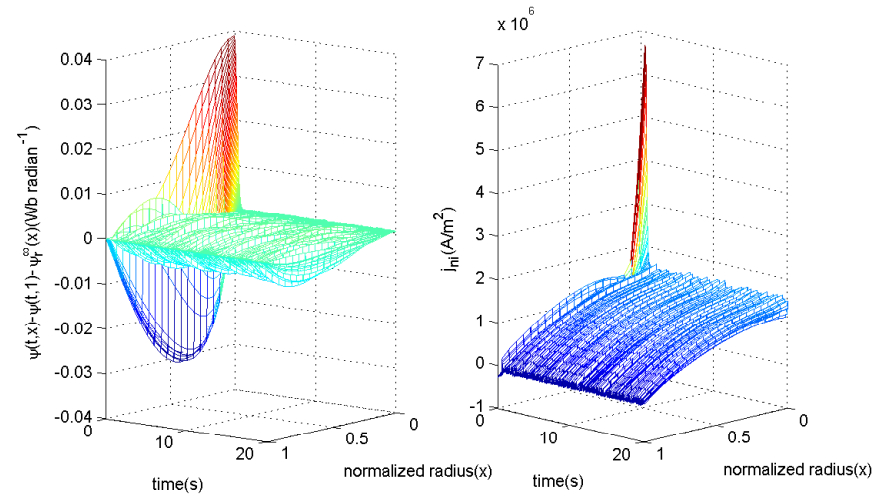

Fig. 7. Evolution of the flux profile error variable $\phi$ and the control $j_{n i}$ : $\mathscr{N}=7.10^{5}, L=10$ and $N=10$ with a disturbance $20 \%$ of $j_{\infty} * \operatorname{rand}(1)$

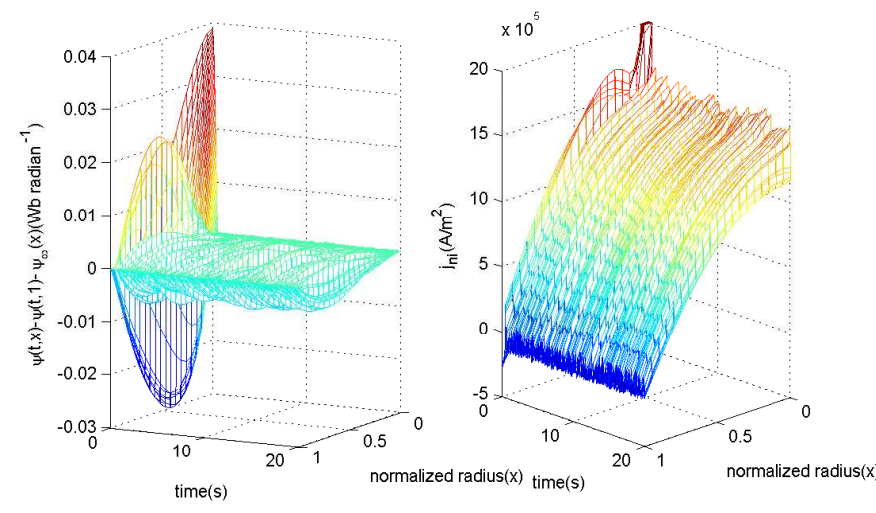

Fig. 8. Evolution of the flux profile error variable $\phi$ and the control $j_{n i}$ : $\mathscr{N}=7.10^{5}, L=10$ and $N=10$ with a disturbance $20 \%$ of $j_{\infty} * \operatorname{rand}(1)$ with saturation

\section{B. Simulation Results}

Simulation results are presented in Figs. 5, 6, 7 and 8. In Fig. 5, time evolution of the flux profile error variable and of the non inductive current density (determine thanks to the sliding mode controller) are presented for the nominal case without perturbation. It is shown that the desired profile is accurately obtained with a proper time delay without overshooting. A numerical scan on the $L$ tuning parameter is performed in order to confirm that a minimum value of $L$ is required in order to guarantee closed loop stability.

In Fig. 6, the evolution of the flux profile error variable and that of the non inductive current density are shown while a constant disturbance is added to $j_{n i}(t, x)$. Considering the previous value of $L$ tuning parameter, a numerical scan on the $\mathscr{N}$ tuning parameter is performed and it is shown that a minimum value of $\mathscr{N}$ is required in order to reject the disturbance.

In Fig. 7, the evolution of the flux profile error variable and that of the non inductive current density are shown for a random (uniform) disturbance added to $j_{n i}(t, x)$ according to (28). As expected for sliding mode control, the system oscillates around the equilibrium.

In Fig. 8, simulation are performed in the same configura- tion except for the control law which is bounded by a realistic value: $\left|j_{n i}(t, x)\right| \leq 2.10^{6} A . m^{-2}$. It is shown that the proposed control strategy answers a proper convergence towards the desired spatial distribution of the magnetic flux.

\section{CONCLUSION AND FUTURE WORK}

In this paper, a sliding mode control in the infinite dimensional setting has been designed for the control of tokamak plasmas current profile using 1D resistive diffusion equation of the magnetic flux. The investigated PDE system was reformulated so as to exhibit a Sturm-Liouville operator. Then recent results on sliding mode control in infinite dimension were applied. First numerical simulations showed consistent results with efficient rejection of disturbances and some "chattering" as expected for sliding mode control.

Several outlooks can be considered. Firstly, numerical simulation have to be performed in order to test situations where plasma resistivity depends on time. Then future works will consist in developing control strategies based on sliding mode considering real engineering control variables, i. e. depending on the tokamak current drive systems : power, phases, or angles that determine the current drive profil deposit.

\section{ACKNOWLEDGMENT}

The authors want to thank Vilmos Komornik (University of Strasbourg-France), Jacques Blum (University of NiceFrance), Christophe Prieur and Emmanuel Witrant (University of Grenoble-France) for fruitful discussion in the preparation of this paper.

\section{REFERENCES}

[1] "www.iter.org."

[2] J. Wesson, Tokamak. Tokamaks 3rd Edition by John Wesson, Oxford University Press, 2004.

[3] B. Saoutic, M. Chatelier, and C. D. Michelis, "Tore supra : toward steady state in a superconducting tokamak," Fusion Science and Technology, vol. 56, no. 3, pp. 1079-1091, 2009.

[4] T. Wijnands, D. V. Haute, G. Martin, X. Litaudon, and P. Froissard, "Feedback control of the current profile on tore supra," Nucl. Fusion, vol. 37, no. 6, pp. 777-791, 1997.

[5] D. Mazon, X. Litaudon, D. Moreau, M. Riva, G. Tresset, Y. Baranov, A. Becoulet, J. M. Chareau, F. Crisanti, R. Dux, R. Felton, E. Joffrin, and contributors to the EFDA-JET workprogramme, "Real time control of internal transport barriers in jet plasma," Phys. Control. Fusion, no. 44, pp. 1087-1104, 2002.

[6] D. Moreau, D. Mazon, M. Ariola, G. D. Tommasi, L. Laborde, F. Piccolo, F. Sartori, T. Tala, L. Zabeo, A. Boboc, E. Bouvier, M. Brix, J. Brzozowski, C. Challis, V. Cocilovo, V. Cordoliani, F. Crisanti, E. D. L. Luna, R. Felton, N. Hawkes, R. King, X. Litaudon, T. Loarer, J. Mailloux, M. Mayoral, I. Nunes, E. Surrey, O. Zimmerman, and J. E. Contributorsa, "A two-scale dynamic model approach for magnetic and kinetic profile control in advanced tokamak scenarios on jet," Nuc. Fusion, vol. 48, no. 10, 2008.

[7] Y. Ou and E. Schuster, "Controllability analysis for current profile control in tokamaks," in IEEE Conference on Decision and Control, Shanghai, China, 2009.

[8] C. Xu, N. J. Dalessio, and E. Schuster, "Optimal control of a parabolic pde system arising in plasma transport via diffusivity interior boundary actuation," in IEEE Conference on Decision and Control, Cancun, Mexico, 2008.

[9] C. Xu, J. Dalessio, Y. Ou, E. Schuster, T. Luce, J. Ferron, M. Walker, and D. Humphreys, "Ramp-up phase current profile control of tokamak plasmas via nonlinear programming," IEEE Transactions on Plasma Science, vol. 38, no. 2, pp. 163-173, 2010. 
[10] J. Blum, Numerical Simulation and Optimal Control in Plasma Phisics With Application to Tokamaks. Wiley / Gauthier-Villars Series in Modern Applied Mathematics, 1989.

[11] E. Witrant, S. Brémond, G. Giruzzi, D. Mazon, O. Barana, and P. Moreau, "A control-oriented model of the current profile in tokamak plasma," Plasma Phys. Control. Fusion, vol. 49, no. 7, pp. 1075-1105, 2007.

[12] A. W. Naylor and G. R. Sell, Linear Operator Theory in Engineering and Science. New York: Springer, 1982.

[13] P. Cannarsa, P. Martinez, and J. Vancostenoble, "Persistent regional controllability for a class of degenerate parabolic equations," Commun. Pure Appl. Anal., vol. 4, no. 3, pp. 607-635, 2004.

[14] P. Cannarsa, G. Fragnelli, and J. Vancostenoble, "Regional controllability of semilinear degenerate parabolic equations in bounded domains," Math. Anal. Appl, vol. 320, no. 2, pp. 804-818, 2006.

[15] L. Rosier, "A survey of controllability and stabilization results for partial differential equations," Journal Européen des Systèmes automatisés, vol. 41, no. 3-4, pp. 365-411, 2007.

[16] Y. Orlov, "Discontinuous unit feedback control of uncertain infinitedimensional systems," IEEE transactions on automatic control, vol. 45, no. 2, pp. 834-843, 2000.

[17] J. M. Coron, Control and Nonlinearity". American Mathematical Society, 2007.

[18] V. Komornik, Exact controllability and stabilization, The Multiplier Method. Masson-John Wiley, 1994, vol. 36.

[19] V. Komornik and P. Loreti, Fourier Series in Control Theory. Springer-Verlag, 2005

[20] P. Christofides, Nolinear and Robust Control of PDE Systems. Birkhuser, 2001

[21] G. P. Menzela and E. Zuazua, "Timoshenkos plate equation as a singular limit of the dynamical von karman system," J.Math. Pures Appl, vol. 79, no. 1, pp. 73-94, 2000.

[22] A. Smyshlyaev and M. Krstic, Adaptive Control of Parabolic PDEs. Princeton University Press, 2010. 